Bull. Austral. Math. Soc.

VOL. 47 (1993) [217-220]

\title{
EQUATIONALLY DEFINED RADICAL CLASSES
}

\author{
N.R. McConnell and Timothy Stokes
}

\begin{abstract}
We consider universal classes of multioperator groups, and give a sufficient condition for a subclass defined by algebraic elementwise rules to be a radical class.
\end{abstract}

Consider a universal class $\mathcal{U}$ of multioperator groups. We obtain a sufficient condition for algebraic element-wise definitions of classes of algebras in $\mathcal{U}$ to give rise to radical classes. Some work of this sort has been done by Gardner [1] and Wiegandt [5]. As our work is largely motivated by rings, we refer to normal subobjects as ideals throughout. We direct the reader to [2] for terminology and background.

DEFINITION 1: Let $\mathcal{U}$ be a universal class in a variety $\mathcal{V}$ of multioperator groups, and let $F$ be a set of elements in the free algebra with countable generators $\left\{x_{1}, x_{2}, \ldots\right\}$ in $\mathcal{V}$. Let $\mathcal{R} F$ be the class in $\mathcal{U}$ defined as follows: $R$ is in $\mathcal{R} F$ providing that for every $r \in R$ there exist $f\left(x_{1}, x_{2}, \ldots, x_{n}\right) \in F$ and $r_{2}, r_{3}, \ldots, r_{n}$ in $R$, such that $f\left(r, r_{2}, r_{3}, \ldots, r_{n}\right)=0$. For any algebra $R$, define $\mathcal{R} F^{\prime}(R)=\{r \in R \mid$ there exists $f \in$ $F$, and $r_{2}, \ldots, r_{n} \in R$ with $\left.f\left(r, r_{2}, \ldots, r_{n}\right)=0\right\}$.

It is obvious from these definitions that $R$ is in $\mathcal{R} F$ if and only if $\mathcal{R} F^{\prime}(R)=R$.

Lemma 2. For all $F, \mathcal{R} F$ is homomorphically closed.

Proof: Suppose $R \in \mathcal{R} F, I \triangleleft R$. If $r+I \in R / I$, then by assumption there exist $f\left(x_{1}, x_{2}, \ldots, x_{n}\right) \in F$ and $r_{2}, r_{3}, \ldots, r_{n} \in R$, such that $f\left(r, r_{2}, r_{3}, \ldots, r_{n}\right)=0$, whence $f\left(r+I, r_{2}+I, r_{3}+I, \ldots, r_{n}+I\right)=0+I$, and so $R / I$ is in $\mathcal{R} F$.

It is shown in [5] that the class of rings whose elements satisfy some property $P$ is a radical class if $P$ satisfies the following conditions (where $I$ is an ideal of some ring A):

(a) If $a$ is a $P$-element of $A$, then the coset $a+I$ is a $P$-element of $A / I$;

(d) If $a$ is a $P$-element in $I$, then $a$ is a $P$-element in $A$ too; and

(e) If the coset $a+I$ is a $P$-element of $A / I$ and $I$ consists of $P$-elements, then $a$ is a $P$-element of $A$.

Received 5th March, 1992.

Copyright Clearance Centre, Inc. Serial-fee code: 0004-9729/93 \$A2.00+0.00. 
We can use this to get a criterion for $\mathcal{R} F$ to be a radical class.

DEFINITION 3: $\quad F$ is $\mathcal{U}$-associating (or just associating when no confusion arises) if and only if for all $R \in \mathcal{U}$, whenever there are $g, h \in F, r, a_{i} \in R$ and $b_{j} \in I$ where $I$ is some ideal of $\mathrm{R}$ with $\mathcal{R} F^{\prime \prime}(I)=I$ for which $g\left(h\left(r, a_{i}\right), b_{j}\right)=0$, there are also $f \in F$ and $c_{k} \in R$ such that $f\left(r, c_{k}\right)=0$. Note that $a_{i}$ here refers to $n-1$ elements of $R$ where $n$ is the number of generators involved in $h$, et cetera.

THEOREM 4. If $F$ is $\mathcal{U}$-associating then $\mathcal{R} F$ is a radical class.

Proof: Suppose $F$ is associating, and let $r$ be a $P$-element of a ring $R$ if $f\left(r, a_{i}\right)=0$ for some $f \in F, a_{i} \in R$. (a) is satisfied by Lemma 2, and (d) is trivial. Suppose that $r+I$ is a $P$-element of $R / I$, where $I$ consists of $P$-elements. Then for some $f \in F$ and $a_{i} \in R, f\left(r+I, a_{i}+I\right)=0$, that is $f\left(r, a_{i}\right) \in I$. Since $I$ consists of $P$-elements, $R F^{\prime}(I)=I$, so there exist $g \in F$ and $b_{j} \in I$ with $g\left(f\left(r, a_{i}\right), b_{j}\right)=0$; hence $h\left(r, c_{k}\right)=0$ for some $h \in F$ as $F$ is associating. Thus $r$ is a $P$-element of $R$, (e) holds, and $\mathcal{R} F$ is a radical class.

Corollary 5. Suppose $F$ is such that $R F^{\prime}(R)$ is an ideal of $R$ for all $R \in \mathcal{U}$, and further that $\mathcal{R} F^{\prime}\left(\mathcal{R} F^{\prime}(R)\right)=\mathcal{R} F^{\prime}(R)$ for all $R \in \mathcal{U}$. Then $\mathcal{R} F$ is a radical class if and only if $F$ is $\mathcal{U}$-associating.

Proof: We show that if $\mathcal{R} F$ is a radical class, then $F$ is associating, the result then following by Theorem 4. Let $R \in \mathcal{U}$. We observe that $\mathcal{R} F^{\prime}(R)$ is the radical of $R$ with respect to $\mathcal{R} F$, since $\mathcal{R} F^{\prime}(R)$ is an ideal of $R$ which obviously contains all other $\mathcal{R} F$-ideals of $R$; moreover, $\mathcal{R} F^{\prime}(R)$ is itself an $\mathcal{R} F$-ideal of $R$ since $\mathcal{R} F^{\prime}\left(\mathcal{R} F^{\prime}(R)\right)=$ $\mathcal{R} F^{\prime}(R)$ by assumption.

Suppose there are $g, h \in F$ and $r, a_{i} \in R, b_{j} \in I$ where $I$ is an ideal of $R$ with $\mathcal{R} F^{\prime}(I)=I$, for which $g\left(h\left(r, a_{i}\right), b_{j}\right)=0$. Then certainly $h\left(r, a_{i}\right)$ is in $\mathcal{R} F^{\prime}(R)$, so that $h\left(r+\mathcal{R} F^{\prime}(R), a_{i}+\mathcal{R} F^{\prime}(R)\right)=0+\mathcal{R} F^{\prime}(R)$. Thus $r+\mathcal{R} F^{\prime}(R) \in \mathcal{R} F^{\prime \prime}\left(R / \mathcal{R} F^{\prime}(R)\right)=0$ whence $r \in \mathcal{R} F(R)$ so that there are $f \in F, c_{k} \in R$ such that $f\left(r, c_{k}\right)=0$ and the result follows.

In this case, $A$ is $\mathcal{R} F$-semisimple if and only if $\mathcal{R} F^{\prime}(A)=\mathcal{R} F(A)=0$ if and only if whenever $f\left(r, a_{i}\right)=0, r=0$.

Corollary 6. Suppose that for all $R \in \mathcal{U}, \mathcal{R} F^{\prime}(R)$ is an ideal of $R$, and that whenever $f\left(r, r_{2}, r_{3}, \ldots, r_{n}\right)=0$ for some $r, r_{2}, \ldots, r_{n} \in R, f \in F$, then all of $r, r_{2}, r_{3}, \ldots, r_{n}$ are in $\mathcal{R} F^{\prime}(R)$. Then $\mathcal{R} F$ is a radical class in $U$ if and only if $F$ is associating.

Proof: Let $R \in \mathcal{U}$. Evidently, for all $r \in \mathcal{R} F^{\prime}(R)$, there exist $r_{2}, r_{3}, \ldots, r_{n} \in$ $\mathcal{R} F^{\prime}(R)$ for which $f\left(r, r_{2}, \ldots, r_{n}\right)=0$. Hence $\mathcal{R} F^{\prime}\left(\mathcal{R} F^{\prime}(R)\right)=\mathcal{R} F^{\prime}(R)$, and the result follows. 
Note that if every $f \in F$ involves only one generator, the second condition of Corollary 6 is satisfied immediately.

Examples. In all the examples below, $\mathcal{U}$ is the class of all associative rings (unless otherwise stated).

(i) Let $F=\{f\}$ where $f(x, y)=x+y+x y$; then $\mathcal{R} F$ is the class of quasiregular rings. Since $f(f(x, y), z)=f(x, y+z+y z)$ for all $x, y, z$ in any ring $R, F$ is associating and by Theorem $4 \mathcal{R} F$ is a radical class (the Jacobson radical class). In fact, $F$ is strongly associating in the sense that for every $f, g \in F$ we have $f\left(g\left(x, y_{i}\right), z_{j}\right)$ actually equal to $h\left(x, p_{k}\left(x, y_{i}, z_{j}\right)\right)$ for some $h \in F$ and polynomials $p_{k}$ in the free algebra. This is also true of all subsequent examples.

(ii) Let $F=\left\{x, x^{2}, \ldots\right\}$; then $\mathcal{R} F$ is the class of nil rings, which is a radical class (the nil radical) because $\left(x^{n}\right)^{m}=x^{n m}$, and so $F$ is associating. Similarly, letting $F=\left\{m x^{n} \mid m, n\right.$ positive integers $\}$ gives the Veldsman radical. Note that if $\mathcal{U}$ is the class of commutative rings, then $\mathcal{R} F^{\prime}(R)$ is an ideal of $R$ for every $R \in \mathcal{U}$, and the remarks after Corollaries 5 and 6 apply. These two are also examples of sets of polynomials closed under substitution, which makes them 1-radicals, where an $n$ radical is a radical class for which a ring $R$ is radical if and only if every $n$-generated subring of $R$ is radical [1, Proposition 4.2].

(iii) In a similar way, all the examples $1-8$ from $[5$, Section 3$]$ arise from strongly associating $F$. It appears possible that all radical classes of the form

$$
\{R \mid \text { every element of } R \text { is a } P \text {-element }\}
$$

arise in this way.

(iv) Let $F_{p}=\{x-p y\}$, where $p$ is prime; then $\mathcal{R} F_{p}$ is the class of $p$-divisible rings, which is a radical class because $(x-p y)-p z=x-p(y+z)$, and so $F_{p}$ is associating. Note that the divisible radical is the intersection of all the $\mathcal{R} F_{p}$ 's.

(v) Let $\mathrm{p}$ and $\mathrm{q}$ be integer polynomials and let $F=\{x-p(x) y q(x)\}$; then $\mathcal{R} F$ is the class of $(p, q)$-regular rings. By an argument similar to that in [3, Lemma 1], $F$ can be shown to be associating.

(vi) Let $\mathcal{U}$ be the class of commutative rings, and let $F=\left\{x^{2 m}+y_{1}{ }^{2}+\cdots+y_{n}{ }^{2} \mid\right.$ $m, n$ positive integers $\}$; then it is fairly easy to see that for any $f_{1}, f_{2} \in F$ there will be $f_{3} \in F$ with $f_{1}\left(f_{2}\left(x, y_{i}\right), v_{j}\right)=f_{3}\left(x, z_{k}\right)$, so $\boldsymbol{F}$ is associating. Note that in this case the conditions of Corollaries 5 and 6 hold. This radical class arises from the real Nullstellensatz of Stengle [4] in the same way that the nil radical arises from the Hilbert Nullstellensatz.

(vii) Let $F=\left\{x^{2}-x\right\}$; then $\mathcal{R} F$ is the class of Boolean rings. $\mathcal{R} F$ is a radical class, but $F$ is not associating. It follows from Corollary 6 that $\mathcal{R} F^{\prime}(R)$ is not always an ideal of $R$; that is, the idempotent elements of a ring do not always form an ideal. 


\section{REFERENCES}

[1] B.J. Gardner, 'Radical properties defined locally by polynomial identities I', J. Austral. Math. Soc. 27 (1979), 257-273.

[2] B.J. Gardner, Radical theory, Pitman Research Notes in Mathematics 198 (Longman Scientific and Technical, Harlow, Essex, UK, 1989).

[3] Gary L. Musser, 'Linear Semiprime (p;q) Radicals', Pacific J. Math. 37 (1971), 749-757.

[4] G. Stengle, 'A Nullstellensatz and a Positivstellensatz in semialgebraic geometry', Math. Ann. 207 (1974), 87-97.

[5] R. Wiegandt, 'Radicals of rings defined by means of elements', Sitz. Ost. Akad. Wiss.II 184 (1975), 117-125.

Department of Mathematics and Computing University of Central Queensland Rockhampton MC Qld 4701 Australia
Department of Mathematics

University of Tasmania

Hobart, Tas 7000

Australia 